Research Article

\title{
Interval Observer Design for One Class of Uncertain Linear Strictly Metzlerian Time-Delay Systems
}

\author{
Dušan Krokavec $(\mathbb{D})$ and Anna Filasová \\ Department of Cybernetics and Artificial Intelligence, Faculty of Electrical Engineering and Informatics, \\ Technical University of Košice, Letná 9, 042 00, Košice, Slovakia
}

Correspondence should be addressed to Dušan Krokavec; dusan.krokavec@tuke.sk

Received 6 November 2020; Revised 27 November 2020; Accepted 3 December 2020; Published 21 December 2020

Academic Editor: Mehmet Emir Koksal

Copyright (c) 2020 Dušan Krokavec and Anna Filasová. This is an open access article distributed under the Creative Commons Attribution License, which permits unrestricted use, distribution, and reproduction in any medium, provided the original work is properly cited.

\begin{abstract}
The paper is concerned with design requirements when the problem of nonnegative state estimation for one class of uncertain linear Metzlerian time-delay systems with constant delays is tackled, while system states take nonnegative values whenever the initial conditions are nonnegative, the upper and lower system matrix bounds are strictly Metzler matrices, and the upper and lower output matrix bounds are nonnegative matrices. By defining positive definite diagonal matrix variables and introducing an associate structure of linear matrix inequalities, the design conditions are proven, guaranteeing if they are feasible, the resulting observer gain matrix is positive and the reflected observer system matrices are strictly Metzler and Hurwitz. A numerical example illustrates the solvability of the proposed design conditions.
\end{abstract}

\section{Introduction}

Positive systems often occur in modeling and control of the class of processes, whose state variables do not have physical meaning unless they are nonnegative $[1,2]$. Since the mathematical theory of Metzler matrices has a close relationship to the theory of positive linear continuous-time dynamical systems, such systems are denoted oftentimes as linear Metzlerian systems. Stability and stabilization of this class of systems are reported, e.g., in [3-5], and related methods, based on linear programming (LP) and linear matrix inequalities (LMIs), are considered for positive stabilization and observer design [6-10]. Admitting the equivalent relation between positive system asymptotic stability and diagonal square stability established in [11], and reflecting a large number of parametric constraints [12], a useful unification is an LMI-based design strategy for linear positive (Metzlerian) systems presented in [13].

Based on nominal system models, the state observers generally asymptotically estimate unknown system state [14]. A counterpart of this approach is outlined in [15] to provide, for given system matrix bounds, the system state estimation in projected intervals. In addition to the above, works on linear interval observer design can be found in this relation (see, e.g., [16-18]), also with the analysis of interval observers grasping the Metzler matrix structures [19]. Interval observer algorithms for uncertain Metzlerian systems, with LMI projection of given interval bounds, are analyzed in $[20]$.

Whilst occurrence of time delays in standard linear systems may lead to substantial degradation of the system stability degree in dependence on delay amplitudes, the stability of delayed positive systems with state affected by time delays does not depend on amplitudes of delays [21]. Especially, it is pointed out that the continuous-time positive systems with time-varying delays are asymptotically stable if the sum of all the system matrices is a Hurwitz matrix. Thus, by applying the theory of positive systems and the theorem of $D$-stability disk region, an interval observer design method for uncertain linear positive systems with time delay is presented in [22].

The main motivation of this paper is to present the design conditions based on strict LMIs, for interval observers related to one class of time-delayed uncertain 
Metzlerian systems. By analyzing the structures of the observer system matrices of strictly Metzler forms, the resulting set of algebraic constraints is formulated as a set of LMIs, conditioned by positive definite diagonal matrix variables, and the stability is forced also through LMIs. Compared with the abovementioned interval observer design methods, the design of interval observers for the considered class of timedelayed uncertain Metzlerian systems becomes much more convenient. Preferring LMI structures, the proofs are of standard way and reflect feasibility of the proposed set of LMIs.

The paper is organized as follows. After Introduction, Section 2 presents some preliminaries, including the characterization of the considered class of time-delay uncertain Metzlerian systems. The main results are presented in Section 3, where the newly introduced set of LMIs, describing the design conditions for the state observer for time-delay linear Metzlerian systems, is theoretically substantiated and proven, and subsequently are generalized conditions for the interval observer design, with application to time-delay uncertain linear Metzlerian systems. To complete the proposed approach, this section presents the basic principle of adaptation to systems with pure Metzler system matrices if the number of columns with zero nondiagonal elements is limited. An example is provided to demonstrate the basic properties of the proposed method in Section 4, while Section 5 draws some conclusions.

Throughout the paper, the following notations are used: $\mathbf{x}^{T}$ and $\mathbf{X}^{T}$ denotes the transpose of the vector $\mathbf{x}$ and the matrix $\mathbf{X}$, respectively, $\operatorname{diag}[\cdot]$ marks a (block) diagonal matrix, $\rho(\mathbf{X})$ indicates eigenvalue spectrum of the square matrix $\mathbf{X}$, for a square symmetric matrix $\mathbf{X} \prec 0$ means that $\mathbf{X}$ is negative definite matrix, the symbol $\mathbf{I}_{n}$ indicates the $n$-th order unit matrix, $\left(\mathbb{R}_{+}\right), \mathbb{R}$ qualifies the set of (nonnegative) real numbers, $\left(\mathbb{R}_{+}^{n \times r}\right), \mathbb{R}^{n \times r}$ refers to the set of $n \times r$ (nonnegative) real matrices, and $\mathbb{M}_{-+}^{n \times n}$ means the set of pure (strictly) Metzler square matrices.

\section{Problem Formulation and Preliminary}

The system class under consideration is multi-input, multioutput (MIMO) linear time-delay Metzler continuoustime dynamic systems, represented in state-space form as follows:

$$
\begin{aligned}
& \dot{q}(t)=\mathbf{A q}(t)+\sum_{i=1}^{p} \mathbf{A}_{i} \mathbf{q}\left(t-\tau_{i}\right)+\mathbf{B u}(t), \\
& \mathbf{y}(t)=\mathbf{C} \mathbf{q}(t)+\sum_{i=1}^{p} \mathbf{C}_{i} \mathbf{q}\left(t-\tau_{i}\right),
\end{aligned}
$$

where the time delays, associated with the system variables, satisfy the condition $0<\tau_{1}<\cdots<\tau_{p} \equiv \tau$ and the system input $\mathbf{u}(t) \in \mathbb{R}^{r}$. The system trajectory $\mathbf{q}(t) \in \mathbb{R}_{+}^{n}$ and the output $\mathbf{y}(t) \in \mathbb{R}_{+}^{m}$ are nonnegative (matrices in the set $\left\{\mathbf{C}, \mathbf{C}_{1}, \ldots, \mathbf{C}_{p} \in \mathbb{R}_{+}^{m \times n}\right\}$ are nonnegative) while matrix $\mathbf{B} \in \mathbb{R}_{+}^{n \times r}$ is nonnegative and $\left\{\mathbf{A}, \mathbf{A}_{1}, \ldots, \mathbf{A}_{p} \in \mathbb{M}_{-+}^{n \times n}\right\}$.
To efficiently introduce the proposed method, some definitions and lemmas, borrowed from the properties of positive systems, are presented at first.

Definition 1. A matrix $\mathbf{Y} \in \mathbb{R}_{+}^{p \times q}$ (a vector $\mathbf{y} \in \mathbb{R}_{+}^{p}$ ) is nonnegative if all its entries are nonnegative and at least one is positive. A matrix $\mathbf{Y} \in \mathbb{R}_{+}^{p \times q}$ (a vector $\mathbf{y} \in \mathbb{R}_{+}^{p}$ ) is positive if all its entries are positive.

Definition 2 (see [23]). A matrix $\mathbf{A} \in \mathbb{R}_{-+}^{n \times n}$ is pure Metzler if its diagonal elements are negative and its off-diagonal elements are nonnegative. A matrix $\mathbf{A} \in \mathbb{R}_{-+}^{n \times n}$ is called strictly Metzler if its diagonal elements are negative and its off-diagonal elements are positive. A Metzler matrix is stable if it is Hurwitz.

Definition 3 (see [24]). Given any nonnegative initial condition $\phi(t) \in \mathbb{R}_{+}^{n}$ such that $\mathbf{q}(t)=\phi(t)$ for $-\tau \leq t \leq 0$, the autonomous part of system (1) is said to be positive if the corresponding trajectory is nonnegative $\left(\mathbf{q}(t) \in \mathbb{R}_{+}^{n}\right.$ for all $\left.t \geq 0\right)$.

Lemma 1 (adapted from [24]). Assuming that the autonomous part of system (1) is asymptotically stable and the matrices $\left\{\mathbf{A}, \mathbf{A}_{1}, \ldots, \mathbf{A}_{p} \in \mathbb{M}_{-+}^{n \times n}\right\}$ are strictly Metzler, then the composed matrix

$$
\mathbf{A}^{\diamond}=\mathbf{A}+\sum_{i=1}^{p} \mathbf{A}_{i},
$$

is strictly Metzler and Hurwitz.

Unless otherwise stated below, the matrices in the set $\left\{\mathbf{A}, \mathbf{A}_{1}, \ldots, \mathbf{A}_{p} \in \mathbb{M}_{-+}^{n \times n}\right\}$ are considered to be strictly Metzler matrices.

Remark 1. If composed matrix (3) is Metzler and Hurwitz, then its transpose is also a Metzler and Hurwitz matrix.

Definition 4 (see [25]). A matrix $\mathbf{L} \in \mathbb{R}^{n \times n}$ is a permutation matrix if exactly one item in each column and row is equal to 1 and all other elements are equal to 0 .

Remark 2. Keeping into account Definition 4 and considering a diagonal matrix $\mathbf{Y} \in \mathbb{R}^{n \times n}$ such that

$$
\mathbf{Y}=\operatorname{diag}\left[y_{1} y_{2}, \ldots, y_{n}\right]
$$

then it yields

$$
\mathbf{L}^{\mathrm{T}} \mathbf{Y} \mathbf{L}=\operatorname{diag}\left[y_{2}, \ldots, y_{n} y_{1}\right]
$$

if $\mathbf{L}^{\mathrm{T}} \in \mathbb{R}^{n \times n}$ takes the circulant form

$$
\mathbf{L}^{\mathrm{T}}=\left[\begin{array}{cc}
\mathbf{0} & \mathbf{I}_{n-1} \\
1 & \mathbf{0}
\end{array}\right] .
$$

Lemma 2 (adapted from [13]). Let the matrix $\mathbf{A} \in \mathbb{M}_{-+}^{n \times n}$ is strictly Metzler, then it is Hurwitz if and only if there exist positive definite diagonal matrices $\mathbf{P}, \mathbf{S} \in \mathbb{R}_{+}^{n \times n}$ such that for $l=1,2, \ldots n, h=1,2, \ldots n-1$, and circulant $\mathbf{L} \in \mathbb{R}_{+}^{n \times n}$, the following sets of linear matrix inequalities are feasible: 
(i)

$$
\begin{gathered}
\mathbf{P} \succ 0, \\
\mathbf{P A}(l, l)_{(1 \leftrightarrow n) / n}<0, \\
\mathbf{P L}^{h} \mathbf{A}(l+h, l)_{(1 \leftrightarrow n) / n} \mathbf{L}^{h \mathrm{~T}}>0, \\
\mathbf{A}^{\mathrm{T}} \mathbf{P}+\mathbf{P A} \prec 0,
\end{gathered}
$$

where

$\mathbf{A}(l+h, l)_{(1 \leftrightarrow n) / n}=\operatorname{diag}\left[a_{1+h, 1}, \ldots, a_{n, n-h} a_{1, n-h+1}, \ldots, a_{h n}\right]$,

(ii)

$$
\begin{gathered}
\mathbf{S} \succ 0, \\
\mathbf{A}(l, l)_{(1 \leftrightarrow n) / n} \mathbf{S}<0, \\
\mathbf{L}^{h} \mathbf{A}(l, l+h)_{(1 \leftrightarrow n) / n} \mathbf{L}^{h \mathrm{~T}} \mathbf{S}>0, \\
\mathbf{S A}^{\mathrm{T}}+\mathbf{A} \mathbf{S} \prec 0,
\end{gathered}
$$

where

$$
\mathbf{A}(l+h, l)_{(1 \leftrightarrow n) / n}=\operatorname{diag}\left[a_{1,1+h}, \ldots, a_{n-h, n} a_{n-h+1,1}, \ldots, a_{n, h}\right] .
$$

Note, LMIs (8) and (13), respectively, reflect structural constraints for elements on the main diagonal of strictly Metzler $\mathbf{A} \in \mathbb{M}_{-+}^{n \times n}$, the set of LMIs (9) and (14), respectively, reflects structural constraints for off-diagonal elements of strictly Metzler $\mathbf{A} \in \mathbb{M}_{-+}^{n \times n}$, and the Lyapunov matrix inequality (10) and (15), respectively, guarantee that $\mathbf{A}$ is Hurwitz, while $\mathbf{P}$ and $\mathbf{S}$, respectively, are positive definite diagonal matrices.

The notation $\Delta=((1 \longleftrightarrow n) / n)$ symbolically expresses that the indexing of the elements in diagonal matrices (11) and (16) is bound to the sum of modulo $n$. We refer the interested reader to [13] and the references therein for further details.

Lemma 3 (Lyapunov inequalities [26]). Consider the delayfree autonomous subsystem

$$
\dot{\mathrm{q}}(t)=\mathbf{A q}(t),
$$

then the following statements are equivalent:

(i) (17) is asymptotically stable.

(ii) $\mathbf{A} \in \mathbb{R}^{n \times n}$ is Hurwitz.

(iii) There exists a symmetric positive definite matrix $\mathbf{P} \in \mathbb{R}^{n \times n}$ satisfying

$$
\mathbf{P}=\mathbf{P}^{\mathrm{T}} \succ 0, \mathbf{P A}+\mathbf{A}^{\mathrm{T}} \mathbf{P} \prec 0 .
$$

(iv) There exists a symmetric positive definite matrix $\mathbf{S} \in \mathbb{R}^{n \times n}$ satisfying

$$
\mathbf{S}=\mathbf{S}^{\mathrm{T}} \succ 0, \mathbf{A} \mathbf{S}+\mathbf{S A}^{\mathrm{T}} \prec 0 .
$$

Note that the equivalent statements (18) and (19) are given to circumvent formulations with inverse matrices since direct use (18) leads to bilinear matrix inequality in the design of the controller and direct use (19) leads to bilinear matrix inequality in the synthesis of the observer. It is easily verifiable that premultiplying the left side and postmultiplying the right side by the matrix $\mathbf{P}=\mathbf{V}^{-1}$ and setting $\mathbf{S}=\mathbf{V}^{-1} \mathbf{U V}^{-1}$, then (18) implies (19).

\section{Main Results}

3.1. State Observer for Time-Delay Metzlerian System. The problem is connected with the existence of the Metzler observer defined for systems (1) and (2) in the form

$$
\begin{aligned}
& \dot{\mathbf{q}}_{e}(t)=\mathbf{A} \mathbf{q}_{e}(t)+\sum_{i=1}^{p} \mathbf{A}_{i} \mathbf{q}_{e}\left(t-\tau_{i}\right)+\mathbf{B u}(t)+\mathbf{J}\left(\mathbf{y}(t)-\mathbf{y}_{e}(t)\right), \\
& \mathbf{y}_{e}(t)=\mathbf{C} \mathbf{q}_{e}(t)+\sum_{i=1}^{p} \mathbf{C}_{i} \mathbf{q}_{e}\left(t-\tau_{i}\right),
\end{aligned}
$$

where $\mathbf{q}_{e}(t) \in \mathbb{R}_{+}^{n}$ is nonnegative estimation of the nonnegative state $\mathbf{q}(t)$ such that the observer error

$$
\mathbf{e}(t)=\mathbf{q}(t)-\mathbf{q}_{e}(t),
$$

converges asymptotically to zero for $t \geq 0$, while $\mathbf{J} \in \mathbb{R}_{+}^{n \times m}$ is positive.

Consequently, by (22) and using (1), (2), (20), and (21), the following yields

$$
\begin{aligned}
\dot{\mathrm{e}}(t) & =(\mathbf{A}-\mathbf{J C}) \mathbf{e}(t)+\sum_{i=1}^{p}\left(\mathbf{A}_{i}-\mathbf{J C}_{i}\right) \mathbf{e}\left(t-\tau_{i}\right), \\
& =\mathbf{A}_{e} \mathbf{e}(t)+\sum_{i=1}^{p} \mathbf{A}_{e i} \mathbf{e}\left(t-\tau_{i}\right),
\end{aligned}
$$

where

$$
\begin{aligned}
\mathbf{A}_{e} & =\mathbf{A}-\sum_{k=1}^{m} \mathbf{j}_{k} \mathbf{c}_{k}^{\mathrm{T}}=\mathbf{A}-\sum_{k=1}^{m} \mathbf{J}_{d k} \mathbf{l}^{\mathrm{T}} \mathbf{C}_{d k}, \\
\mathbf{A}_{e i} & =\mathbf{A}_{i}-\sum_{k=1}^{m} \mathbf{j}_{k} \mathbf{c}_{i k}^{\mathrm{T}}=\mathbf{A}_{i}-\sum_{k=1}^{m} \mathbf{J}_{d k} \mathbf{l}^{\mathrm{T}} \mathbf{C}_{d i k}, \\
\mathbf{C} & =\left[\begin{array}{c}
\mathbf{c}_{1}^{\mathrm{T}} \\
\vdots \\
\mathbf{c}_{m}^{\mathrm{T}}
\end{array}\right], \mathbf{C}_{d k}=\operatorname{diag}\left[\mathbf{c}_{k}^{\mathrm{T}}\right]=\operatorname{diag}\left[\begin{array}{lll}
c_{k 1} & \cdots & c_{k n}
\end{array}\right], \\
\mathbf{C}_{i} & =\left[\begin{array}{c}
\mathbf{c}_{i 1}^{\mathrm{T}} \\
\vdots \\
\mathbf{c}_{i m}^{\mathrm{T}}
\end{array}\right], \mathbf{C}_{d i k}=\operatorname{diag}\left[\begin{array}{c}
c_{i k}^{\mathrm{T}}
\end{array}\right]=\operatorname{diag}\left[\begin{array}{lll}
c_{i k 1} & \cdots & c_{i k n}
\end{array}\right],
\end{aligned}
$$




$$
\begin{aligned}
\mathbf{J} & =\left[\mathbf{j}_{1}, \ldots, \mathbf{j}_{m}\right], \mathbf{J}_{d k}=\operatorname{diag}\left[\mathbf{j}_{k}\right]=\operatorname{diag}\left[j_{k 1}, \ldots, j_{k n}\right], \\
\mathbf{l}^{\mathrm{T}} & =[11, \ldots, 1],
\end{aligned}
$$

and (3) implies that

$$
\mathbf{A}_{e}^{\diamond}=\mathbf{A}_{e}+\sum_{i=1}^{p} \mathbf{A}_{e i}
$$

is Hurwitz.

Theorem 1. The matrix $\mathbf{A}_{e}$ is strictly Metzler and Hurwitz and the matrix $\mathbf{A}_{e}^{\diamond}$ is pure Hurwitz if for given strictly Metzler $\mathbf{A}, \mathbf{A}_{1}, \ldots, \mathbf{A}_{p} \in \mathbb{M}_{-+}^{n \times n}$ and nonnegative $\mathbf{C}, \mathbf{C}_{1}, \ldots, \mathbf{C}_{p} \in \mathbb{R}_{+}^{m \times n}$ there exist positive definite diagonal matrices $\mathbf{P}, \mathbf{Q} \in \mathbb{R}_{+}^{n \times n}$ such that for $l=1,2, \ldots, n, \quad h=1,2, \ldots, n-1$, $k=1,2, \ldots, m$, and the circulant $\mathbf{L} \in \mathbb{R}_{+}^{n \times n}$, the following set of linear matrix inequalities is feasible:

$$
\begin{aligned}
& \mathbf{P}>0, \mathbf{R}_{k}>0, \\
& \mathbf{P A}(l, l)_{(1 \leftrightarrow n) / n}-\sum_{k=1}^{m} \mathbf{R}_{k} \mathbf{C}_{d k} \prec 0, \\
& \mathbf{P} \mathbf{L}^{h} \mathbf{A}(l+h, l)_{(1 \leftrightarrow n) / n} \mathbf{L}^{h T}-\sum_{k=1}^{m} \mathbf{R}_{k} \mathbf{L}^{h} \mathbf{C}_{d k} \mathbf{L}^{h T}>0, \\
& {\left[\begin{array}{cccc}
\mathbf{P A}+\mathbf{A}^{\mathrm{T}} \mathbf{P}-\sum_{k=1}^{m} \mathbf{R}_{k} \mathbf{l l}^{\mathrm{T}} \mathbf{C}_{d k}-\sum_{k=1}^{m} \mathbf{C}_{d k} \mathbf{l l}^{\mathrm{T}} \mathbf{R}_{k} & * & \cdots & * \\
\mathbf{P} \mathbf{A}_{1}-\sum_{k=1}^{m} \mathbf{R}_{k} \mathbf{l l}^{\mathrm{T}} \mathbf{C}_{d 1 k} & -\mathbf{Q} & \cdots & * \\
\vdots & \vdots & \ddots & \vdots \\
\mathbf{P A}_{p}-\sum_{k=1}^{m} \mathbf{R}_{k} \mathbf{l l}^{\mathrm{T}} \mathbf{C}_{d p k} & \mathbf{0} & \cdots & -\mathbf{Q}
\end{array}\right] \prec 0 .}
\end{aligned}
$$

When the above conditions hold, the positive observer gain $\mathbf{J} \in \mathbb{R}_{+}^{n \times m}$ is given as

$$
\mathbf{J}_{d k}=\mathbf{P}^{-1} \mathbf{R}_{k}, \mathbf{j}_{k}=\mathbf{J}_{d k} \mathbf{l}, \mathbf{J}=\left[\mathbf{j}_{1}, \ldots, \mathbf{j}_{m}\right] .
$$

Hereafter, * denotes the symmetric item in a symmetric matrix.

Proof. Defining with positive definite diagonal matrices $\mathbf{P}, \mathbf{Q} \in \mathbb{R}_{+}^{n \times n}$, the Lyapunov function

$$
v(\mathbf{e}(t))=\mathbf{e}^{T}(t) \mathbf{P e}(t)+\sum_{i=1}^{p} \int_{t-\tau_{i}}^{t} \mathbf{e}^{T}(v) \mathbf{Q e}(v) \mathrm{d} v,
$$

it follows from (34)

$$
\begin{aligned}
\dot{v}(\mathbf{e}(t))= & \dot{\mathrm{e}}^{\mathrm{T}}(t) \mathbf{P e}(t)+\mathbf{e}^{\mathrm{T}}(t) \mathbf{P} \dot{\mathrm{e}}(t)+p \mathbf{e}^{\mathrm{T}}(t) \mathbf{Q} \mathbf{e}(t)-, \\
& -\sum_{k=1}^{m} \mathbf{e}^{T}\left(t-\tau_{i}\right) \mathbf{Q} \mathbf{e}\left(t-\tau_{i}\right) .
\end{aligned}
$$

Using a standard way, relation (35) can be rewritten as follows:

$$
\begin{aligned}
& \dot{\mathrm{e}}^{\mathrm{T}}(t) \mathbf{P e}(t)+\mathbf{e}^{\mathrm{T}}(t) \mathbf{P} \dot{\mathrm{e}}(t)-\sum_{k=1}^{m} \mathbf{e}^{\mathrm{T}}\left(t-\tau_{i}\right) \mathbf{Q} \mathbf{e}\left(t-\tau_{i}\right) \\
& <-p \mathbf{e}^{\mathrm{T}}(t) \mathbf{Q} \mathbf{e}(t)<0,
\end{aligned}
$$

and because for a stable Metzler observer the product $\mathbf{e}^{\mathrm{T}}(t) \mathbf{Q} \mathbf{e}(t)$ is positive and converges to zero at time $t \longrightarrow \infty$, the linearity allows to consider that the function on the left side of (36) must be negative definite and it must converge for $t \longrightarrow \infty$ from the left to zero. Thus, the left side of (36) can be represented as

$$
\mathbf{e}^{\circ \mathrm{T}}(t) \mathbf{P}^{\circ} \mathbf{e}^{\circ}(t)<0,
$$

where

$$
\begin{aligned}
\mathbf{e}^{\circ \mathrm{T}}(t) & =\left[\mathbf{e}^{\mathrm{T}}(t) \mathbf{e}^{\mathrm{T}}\left(t-\tau_{1}\right), \ldots, \mathbf{e}^{\mathrm{T}}\left(t-\tau_{p}\right)\right], \\
\mathbf{P}^{\circ} & =\left[\begin{array}{cccc}
\mathbf{P} \mathbf{A}_{e}+\mathbf{A}_{e}^{\mathrm{T}} \mathbf{P} & \mathbf{A}_{e 1}^{\mathrm{T}} \mathbf{P} & \cdots & \mathbf{A}_{e p}^{\mathrm{T}} \mathbf{P} \\
\mathbf{P} \mathbf{A}_{e 1} & -\mathbf{Q} & \cdots & \mathbf{0} \\
\vdots & \vdots & \ddots & \vdots \\
\mathbf{P A}_{e p} & \mathbf{0} & \cdots & -\mathbf{Q}
\end{array}\right] \prec 0 .
\end{aligned}
$$

Since the following implications hold from (24)

$$
\begin{gathered}
\mathbf{P A}_{e}=\mathbf{P A}-\sum_{k=1}^{m} \mathbf{P} \mathbf{J}_{d k} \mathbf{l l}^{\mathrm{T}} \mathbf{C}_{d k}=\mathbf{P A}-\sum_{k=1}^{m} \mathbf{R}_{k} \mathbf{l l}^{\mathrm{T}} \mathbf{C}_{d k}, \\
\mathbf{P} \mathbf{A}_{e i}=\mathbf{P A}-\sum_{k=1}^{m} \mathbf{P} \mathbf{J}_{d k} \mathbf{l l}^{\mathrm{T}} \mathbf{C}_{d i k}=\mathbf{P} \mathbf{A}_{i}-\sum_{k=1}^{m} \mathbf{R}_{k} \mathbf{l l}^{\mathrm{T}} \mathbf{C}_{d i k},
\end{gathered}
$$

where

$$
\mathbf{R}_{k}=\mathbf{P} \mathbf{J}_{d k},
$$

then (39) conditioned by (40)-(42) implies (32).

According to the definition, $\mathbf{A}_{e}$ is strictly Metzler if for $l, j=1,2, \ldots, n$,

$$
a_{l l}-\sum_{k=1}^{m} j_{k l} c_{k l}<0, a_{l j}-\sum_{k=1}^{m} j_{k l} c_{k j}>0, \quad l \neq j .
$$

In relation with (8) and (9), constraint inequalities (43) determine the relations [13]

$$
\mathbf{A}(l, l)_{(1 \leftrightarrow n)}-\sum_{k=1}^{m} \mathbf{J}_{d k} \mathbf{C}_{d k} \prec 0,
$$

$$
\mathbf{L}^{h} \mathbf{A}(l+h, l)_{(1 \leftrightarrow n) / n} \mathbf{L}^{h \mathrm{~T}}-\sum_{k=1}^{m} \mathbf{J}_{d k} \mathbf{L}^{h} \mathbf{C}_{d k} \mathbf{L}^{h \mathrm{~T}}>0 .
$$


Thus, multiplying the left sides of (44) and (45) by $\mathbf{P}$, it yields

$$
\begin{array}{r}
\mathbf{P A}(l, l)_{(1 \leftrightarrow n)}-\sum_{k=1}^{m} \mathbf{P} \mathbf{J}_{d k} \mathbf{C}_{d k}>0, \\
\mathbf{P} \mathbf{L}^{h} \mathbf{A}(l+h, l)_{(1 \leftrightarrow n) / n} \mathbf{L}^{h \mathrm{~T}}-\sum_{k=1}^{m} \mathbf{P} \mathbf{J}_{d k} \mathbf{L}^{h} \mathbf{C}_{d k} \mathbf{L}^{h \mathrm{~T}}>0,
\end{array}
$$

and inserting (42), then (46) and (47) implies (30) and (31), respectively.

Using the Schur complement property, inequality (39) can be rewritten as follows:

$$
\mathbf{P A}_{e}+\mathbf{A}_{e}^{\mathrm{T}} \mathbf{P}+\left[\begin{array}{lll}
\mathbf{A}_{e 1}^{\mathrm{T}} \mathbf{P} & \cdots & \mathbf{A}_{e p}^{\mathrm{T}} \mathbf{P}
\end{array}\right]\left[\begin{array}{ccc}
\mathbf{Q}^{-1} & \cdots & \mathbf{0} \\
\vdots & \ddots & \vdots \\
\mathbf{0} & \cdots & \mathbf{Q}^{-1}
\end{array}\right]\left[\begin{array}{c}
\mathbf{P A}_{e 1} \\
\vdots \\
\mathbf{P A}_{e p}
\end{array}\right] \prec 0 .
$$

Then, the second matrix in (48) is positive definite. Applying conversely Lemma 3 then, since $\mathbf{P}$ is positive definite and it satisfied the inequality

$$
\mathbf{P} \mathbf{A}_{e}+\mathbf{A}_{e}^{\mathrm{T}} \mathbf{P}=\mathbf{P A}+\mathbf{A}^{\mathrm{T}} \mathbf{P}-\sum_{k=1}^{m} \mathbf{R}_{k} \mathbf{l l}^{\mathrm{T}} \mathbf{C}_{d k}-\sum_{k=1}^{m} \mathbf{C}_{d k} \mathbf{l}^{\mathrm{T}} \mathbf{R}_{k} \prec 0
$$

then $\mathbf{A}_{e}$ is stable.

Therefore, if inequality (32) is negative definite, also (49) is negative definite and Lyapunov matrix inequality property implies that $\mathbf{A}_{e}$ is Hurwitz. Moreover, (30) and (31) guarantee that $\mathbf{A}_{e}$ is Metzler, while $\mathbf{A}_{e}^{\circ}$ is Hurwitz since convex combination of stable Metzler matrices is Hurwitz [27]. This completes the proof.

Theorem 2. The matrices $\mathbf{A}_{e}, \mathbf{A}_{e}^{\diamond}$ as well as the set of matrices $\left\{\mathbf{A}_{e i}, i=1, \ldots, p\right\}$ are strictly Metzler and Hurwitz, if for given strictly Metzler $\mathbf{A}, \mathbf{A}_{1}, \ldots, \mathbf{A}_{p} \in \mathbb{M}_{-+}^{n \times n}$ and nonnegative $\mathbf{C}, \mathbf{C}_{1}, \ldots, \mathbf{C}_{p} \in \mathbb{R}_{+}^{m \times n}$ there exist positive definite diagonal matrices $\mathbf{P}, \mathbf{Q} \in \mathbb{R}_{+}^{n \times n}$ such that for $l=1,2, \ldots, n$, $h=1,2, \ldots n-1, k=1,2, \ldots m, i=1,2, \ldots, p$, and the circulant $\mathbf{L} \in \mathbb{R}_{+}^{n \times n}$ the following set of linear matrix inequalities is feasible

$$
\begin{array}{r}
\mathbf{P} \succ 0, \mathbf{R}_{k} \succ 0, \\
\mathbf{P A}(l, l)_{(1 \leftrightarrow n) / n}-\sum_{k=1}^{m} \mathbf{R}_{k} \mathbf{C}_{d k} \prec 0, \\
\mathbf{P L}^{h} \mathbf{A}(l+h, l)_{(1 \leftrightarrow n) / n} \mathbf{L}^{h \mathrm{~T}}-\sum_{k=1}^{m} \mathbf{R}_{k} \mathbf{L}^{h} \mathbf{C}_{d k} L^{h \mathrm{~T}} \succ 0, \\
\mathbf{P A}_{i}(l, l)_{(1 \leftrightarrow n) / n}-\sum_{k=1}^{m} \mathbf{R}_{k} \mathbf{C}_{d i k} \prec 0,
\end{array}
$$

$$
\begin{gathered}
\mathbf{P} \mathbf{L}^{h} \mathbf{A}_{i}(l+h, l)_{(1 \leftrightarrow n) / n} \mathbf{L}^{h T}-\sum_{k=1}^{m} \mathbf{R}_{k} \mathbf{L}^{h} \mathbf{C}_{d i k} \mathbf{L}^{h \mathrm{~T}}>0, \\
{\left[\begin{array}{cccc}
\mathbf{P A}+\mathbf{A}^{\mathrm{T}} \mathbf{P}-\sum_{k=1}^{m} \mathbf{R}_{k} \mathbf{l l}^{\mathrm{T}} \mathbf{C}_{d k}-\sum_{k=1}^{m} \mathbf{C}_{d k} \mathbf{l l}^{\mathrm{T}} \mathbf{R}_{k} & * & \cdots & * \\
\mathbf{P} \mathbf{A}_{1}-\sum_{k=1}^{m} \mathbf{R}_{k} \mathbf{l l}^{\mathrm{T}} \mathbf{C}_{d 1 k} & -\mathbf{Q} & \cdots & * \\
\vdots & \vdots & \ddots & \vdots \\
\mathbf{P A}_{p}-\sum_{k=1}^{m} \mathbf{R}_{k} \mathbf{l l}^{\mathrm{T}} \mathbf{C}_{d p k} & 0 & \cdots & -\mathbf{Q}
\end{array}\right]<0 .}
\end{gathered}
$$

When the above conditions hold, the positive observer gain $\mathbf{J} \in \mathbb{R}_{+}^{n \times m}$ is given as in (33).

Proof. Expanding the design conditions (29)-(32) by the set of matrix inequalities (52) and (53) to the extended set (50)-(55), then it forces that all $\mathbf{A}_{e i}$ will have a strictly Metzler structure within feasible (50)-(55). Moreover, because the sum of strictly Metzler matrices is a strictly Metzler matrix, $\mathbf{A}_{e}^{\diamond}$ will be strictly Metzler. Therefore, (48) can be rewritten as follows:

$$
\mathbf{P} \mathbf{A}_{e}+\mathbf{A}_{e}^{\mathrm{T}} \mathbf{P}+\sum_{i=1}^{p} \mathbf{A}_{e i}^{\mathrm{T}} \mathbf{P} \mathbf{Q}^{-1} \mathbf{P} \mathbf{A}_{e i} \prec 0 .
$$

Since $\mathbf{P}, \mathbf{Q} \in \mathbb{R}_{+}^{n \times n}$ are positive definite diagonal matrices, it can be written for $i=s, s \in\langle 1, p\rangle$ as

$$
\left(\mathbf{A}_{e s}^{\mathrm{T}} \mathbf{P}-\mathbf{I}_{n}\right) \mathbf{Q}^{-1}\left(\mathbf{P} \mathbf{A}_{e s}-\mathbf{I}_{n}\right)>0,
$$

where $\left(\mathbf{P A}_{e s}-\mathbf{I}_{n}\right)$ and $\left(\mathbf{A}_{e s}^{\mathrm{T}} \mathbf{P}-\mathbf{I}_{n}\right)$ are also strictly Metzler. This is the same as

$$
\begin{array}{r}
\mathbf{Q}^{-1}+\mathbf{A}_{e s}^{\mathrm{T}} \mathbf{P} \mathbf{Q}^{-1} \mathbf{P} \mathbf{A}_{e s}-\mathbf{A}_{e i}^{\mathrm{T}} \mathbf{P} \mathbf{Q}^{-1}-\mathbf{Q}^{-1} \mathbf{P} \mathbf{A}_{e s}>0, \\
-\left(\mathbf{Q}^{-1}+\mathbf{A}_{e s}^{\mathrm{T}} \mathbf{P} \mathbf{Q}^{-1} \mathbf{P} \mathbf{A}_{e s}\right)+\mathbf{A}_{e s}^{\mathrm{T}} \mathbf{P} \mathbf{Q}^{-1}+\mathbf{Q}^{-1} \mathbf{P} \mathbf{A}_{e s} \prec 0,
\end{array}
$$

respectively, and (59) is satisfied if

$$
\mathbf{A}_{e s}^{\mathrm{T}} \mathbf{P} \mathbf{Q}^{-1}+\mathbf{Q}^{-1} \mathbf{P} \mathbf{A}_{e s} \prec 0 .
$$

Thus, since $\mathbf{P}, \mathbf{Q} \in \mathbb{R}_{+}^{n \times n}$ are positive definite diagonal matrices, $\mathbf{A}_{e s}$ is Metzler and (60) is negative definite; Lemma 3 implies that the matrix $\mathbf{A}_{e s}$ is Hurwitz.

Thus, within the above design procedure, all $\mathbf{A}_{e i}$ are fixed to be strictly Metzler and Hurwitz. This concludes the proof.

Corollary 1. Consider that $\mathbf{A}, \mathbf{A}_{1}, \ldots, \mathbf{A}_{p} \in \mathbb{M}_{-+}^{n \times n}$ are pure Metzler but all mutually compatible in the position of a zero off-diagonal column elements (zero element falls in all these matrices on column number $z$ that is $(*, z)$-elements $=0$, $* \neq z)$ and that $\mathbf{C}, \mathbf{C}_{1}, \ldots, \mathbf{C}_{p} \in \mathbb{R}_{+}^{m \times n}$ are nonnegative matrices in all zero element positions mutually compatible.

If the $z$-th column in zero mutually compatible set $\mathbf{C}, \mathbf{C}_{1}, \ldots, \mathbf{C}_{p}$ is zero vector that is $\mathbf{c}_{z}=\mathbf{c}_{i z}=\mathbf{0}$, the design conditions defined by Theorem 2 remain valid, and it results positive $\mathbf{J}$ and pure Metzler and Hurwitz matrices $\mathbf{A}_{e}, \mathbf{A}_{e 1}, \ldots, \mathbf{A}_{e p}, \mathbf{A}_{e}^{\diamond} \in \mathbb{M}_{-+}^{n \times n}$. 
If all elements of the $z$-th column in zero mutually compatible set $\mathbf{C}, \mathbf{C}_{1}, \ldots, \mathbf{C}_{p}$ are nonzero and $(x, z)$-element in mutually zero compatible set $\mathbf{A}, \mathbf{A}_{1}, \ldots, \mathbf{A}_{p} \in \mathbb{M}_{-+}^{n \times n}$ is equal to zero, and the design conditions defined by Theorem 2 remain valid but with the structured matrix variables

$$
\mathbf{R}_{k}=\operatorname{diag}\left[r_{k 1}, \ldots, r_{k, z-1} r_{k x} r_{k, z+1}, \ldots, r_{k n}\right],
$$

where

$$
r_{k x}=0, \quad r_{k z}>0 \text { for } x \neq z, z=1, \ldots, n, k=1, \ldots, m,
$$

and the results form a nonnegative $\mathbf{J}$ and pure Metzler and Hurwitz observer matrices $\mathbf{A}_{e}, \mathbf{A}_{e 1}, \ldots, \mathbf{A}_{e p}, \mathbf{A}_{e}^{\diamond} \in \mathbb{M}_{-+}^{n \times n}$.

All other cases can be defined "ad hoc," limiting in $k$ in (61) with dependency on the structure of nonzero elements in $\mathbf{c}_{z}=\mathbf{c}_{i z}$ or limiting in $i$ in (53) and (54) with dependency on off-diagonal zero elements in $\mathbf{A}, \mathbf{A}_{i}$, or using design conditions defined in Theorem 1 . The latter possibilities may mean that some $\mathbf{A}_{e i}$ will be Hurwitz but not Metzler.

The proofs are closely related to that one presented in Theorem 2.

Remark 3. If there is at least one zero off-diagonal element in each column of matrix $\mathbf{A}$, a solution for a pure Metzler and Hurwitz $\mathbf{A}_{e}$ generally requires that matrix $\mathbf{C}$ be a signum indefinite. By analogy, it can be generalized to pairs $\left(\mathbf{A}_{i}, \mathbf{C}_{i}\right)$ (see [7]).

3.2. Interval Observer for Time-Delay Uncertain Metzlerian System. Consider (1) and (2), where time delays $0<\tau_{1}<\cdots<\tau_{p} \equiv \tau$ are constant, while $(\mathbf{A}, \mathbf{C}),\left(\mathbf{A}_{i}, \mathbf{C}_{i}\right)$, and $\mathbf{q}(0)$ are unknown but bounded, and for all $i \in\langle 1, p\rangle$, the known constant bounds satisfy elementwise the conditions

$$
\begin{aligned}
\underline{\mathbf{q}}(0) & \leq \mathbf{q}(0) \leq \overline{\mathbf{q}}(0), \underline{\mathbf{A}} \leq \mathbf{A} \leq \overline{\mathbf{A}}, \underline{\mathbf{A}}_{i} \leq \mathbf{A}_{\mathbf{i}} \leq \overline{\mathbf{A}}_{i}, \underline{\mathbf{C}} \leq \mathbf{C} \leq \overline{\mathbf{C}}, \underline{\mathbf{C}}_{i} \\
& \leq \mathbf{C}_{i} \leq \overline{\mathbf{C}}_{i},
\end{aligned}
$$

where $\overline{\mathbf{A}}, \underline{\mathbf{A}} \in \mathbb{M}_{-+}^{n \times n}$ and $\overline{\mathbf{A}}_{i}, \underline{\mathbf{A}}_{i} \in \mathbb{M}_{-+}^{n \times n}$ for all $i \in\langle 1, p\rangle$ are strictly Metzler and $\overline{\mathbf{C}}, \underline{\mathbf{C}} \in \mathbb{R}_{+}^{m \times n}$ and $\overline{\mathbf{C}}_{i}, \underline{\mathbf{C}}_{i} \in \mathbb{R}_{+}^{\times n}$ for all $i \in\langle 1, p\rangle$ are nonnegative.

The aforementioned problem is turned to construction of (strictly) Metzlerian interval observer, defined by the following couple of algorithms:

$$
\dot{\overline{\mathrm{q}}}_{e}(t)=\overline{\mathbf{A}} \overline{\mathbf{q}}_{e}(t)+\sum_{i=1}^{p} \overline{\mathbf{A}}_{i} \overline{\mathbf{q}}_{e}\left(t-\tau_{i}\right)+\mathbf{B u}(t)+\mathbf{J}\left(\mathbf{y}(t)-\underline{\mathbf{y}}_{e}(t)\right),
$$

$$
\underline{\mathrm{q}}_{e}(t)=\underline{\mathbf{A}}_{\underline{\mathbf{q}}_{e}}(t)+\sum_{i=1}^{p} \underline{\mathbf{A}}_{i} \overline{\mathbf{q}}_{e}\left(t-\tau_{i}\right)+\mathbf{B}_{i} \mathbf{u}(t)+\mathbf{J}\left(\mathbf{y}(t)-\overline{\mathbf{y}}_{e}(t)\right),
$$

where

$$
\begin{aligned}
& \overline{\mathbf{y}}_{e}(t)=\overline{\mathbf{C}} \overline{\mathbf{q}}_{e}(t)+\sum_{i=1}^{p} \overline{\mathbf{C}}_{i} \overline{\mathbf{q}}_{e}\left(t-\tau_{i}\right), \\
& \underline{\mathbf{y}}_{e}(t)=\underline{\mathbf{C}} \underline{\mathbf{q}}_{e}(t)+\sum_{i=1}^{p} \underline{\mathbf{C}}_{i} \underline{\mathbf{q}}_{e}\left(t-\tau_{i}\right),
\end{aligned}
$$

and the design objective constraints are stated as

$$
\mathbf{0} \leq \underline{\mathbf{q}}_{e}(t) \leq \mathbf{q}(t) \leq \overline{\mathbf{q}}_{e}(t) .
$$

The set of equations (64)-(67) give a stable interval observer for uncertain Metzlerian plant (1) and (2) if both the lower estimation error $\mathbf{e}(t)$ and the upper estimation error $\overline{\mathbf{e}}(t)$ defined as

$$
\overline{\mathbf{e}}(t)=\mathbf{q}(t)-\overline{\mathbf{q}}_{e}(t), \underline{\mathbf{e}}(t)=\mathbf{q}(t)-\underline{\mathbf{q}}_{e}(t),
$$

converge to the equilibrium.

Assumption 1. If (1), (2), and (64)-(67) are rearranged as

$$
\begin{aligned}
\dot{\overline{\mathbf{e}}}(t) & =(\overline{\mathbf{A}}-\mathbf{J} \underline{\mathbf{C}}) \overline{\mathbf{e}}(t)+\sum_{i=1}^{p}\left(\overline{\mathbf{A}}_{i}-\mathbf{J} \underline{\mathbf{C}}_{i}\right) \overline{\mathbf{e}}\left(t-\tau_{i}\right), \\
& =\overline{\mathbf{A}}_{e} \overline{\mathbf{e}}(t)+\sum_{i=1}^{p} \overline{\mathbf{A}}_{e i} \overline{\mathbf{e}}\left(t-\tau_{i}\right), \\
\underline{\mathbf{e}}(t) & =(\underline{\mathbf{A}}-\mathbf{J} \overline{\mathbf{C}}) \underline{\mathbf{e}}(t)+\sum_{i=1}^{p}\left(\underline{\mathbf{A}}_{i}-\mathbf{J} \overline{\mathbf{C}}_{i}\right) \underline{\mathbf{e}}\left(t-\tau_{i}\right), \underline{\mathbf{A}_{e}} \underline{\mathbf{e}}(t)+\sum_{i=1}^{p} \underline{\mathbf{A}}_{e i} \underline{\mathbf{e}}\left(t-\tau_{i}\right),
\end{aligned}
$$


where

$$
\begin{aligned}
\overline{\mathbf{A}}_{e} & =\overline{\mathbf{A}}-\mathbf{J} \underline{\mathbf{C}}=\overline{\mathbf{A}}-\sum_{k=1}^{m} \mathbf{j}_{k} \underline{\mathbf{c}}_{k}^{\mathrm{T}}=\overline{\mathbf{A}}-\sum_{k=1}^{m} \mathbf{J}_{d k} \mathbf{l l}^{\mathrm{T}} \underline{\mathbf{C}}_{d k}, \\
\underline{\mathbf{A}}_{e i} & =\underline{\mathbf{A}}-\mathbf{J} \overline{\mathbf{C}}=\underline{\mathbf{A}}_{i}-\sum_{k=1}^{m} \mathbf{j}_{k} \overline{\mathbf{c}}_{i k}^{\mathrm{T}}=\overline{\mathbf{A}}_{i}-\sum_{k=1}^{m} \mathbf{J}_{d k} \mathbf{l l}^{\mathrm{T}} \underline{\mathbf{C}}_{d i k}, \\
\overline{\mathbf{C}} & =\left[\begin{array}{c}
\overline{\mathbf{c}}_{1}^{\mathrm{T}} \\
\vdots \\
\overline{\mathbf{c}}_{m}^{\mathrm{T}}
\end{array}\right], \underline{\mathbf{C}}=\left[\begin{array}{c}
\underline{\mathbf{c}}_{1}^{\mathrm{T}} \\
\vdots \\
\underline{\mathbf{c}}_{m}^{\mathrm{T}}
\end{array}\right], \overline{\mathbf{C}}_{i}=\left[\begin{array}{c}
\overline{\mathbf{c}}_{i 1}^{\mathrm{T}} \\
\vdots \\
\overline{\mathbf{c}}_{i m}^{\mathrm{T}}
\end{array}\right], \underline{\mathbf{C}}_{i}=\left[\begin{array}{c}
\underline{\mathbf{c}}_{i 1}^{\mathrm{T}} \\
\vdots \\
\underline{\mathbf{c}}_{i m}^{\mathrm{T}}
\end{array}\right], \\
\overline{\mathbf{C}}_{d k} & =\operatorname{diag}\left[\overline{\boldsymbol{c}}_{k 1}, \ldots, \overline{\boldsymbol{c}}_{k n}\right], \underline{\mathbf{C}}_{d k}=\operatorname{diag}\left[\underline{c}_{k 1}, \ldots, \underline{c}_{k n}\right], \\
\overline{\mathbf{C}}_{d i k} & =\operatorname{diag}\left[\bar{c}_{i k 1}, \ldots, \bar{c}_{i k n}\right], \underline{\mathbf{C}}_{d i k}=\operatorname{diag}\left[\underline{c}_{i k 1}, \ldots, \underline{c}_{i k n}\right],
\end{aligned}
$$

and $\mathbf{J}, \mathbf{l}^{\mathrm{T}}$ are defined in (26) and (27), then (70) and (71) are asymptotically stable if for pair sets $(\overline{\mathbf{A}}, \underline{\mathbf{A}}),(\overline{\mathbf{C}}, \underline{\mathbf{C}}),\left(\overline{\mathbf{A}}_{i}, \underline{\mathbf{A}}_{i}\right)$,
$\left(\overline{\mathbf{C}}_{i}, \underline{\mathbf{C}}_{i}\right)$ satisfying (63) the resulting matrices $\overline{\mathbf{A}}_{e}, \overline{\mathbf{A}}_{e}^{\diamond}, \underline{\mathbf{A}}_{e}, \underline{\mathbf{A}}_{e}^{\diamond}$, $\overline{\mathbf{A}}_{e i}, \underline{\mathbf{A}}_{e i}$ are strictly Metzler and Hurwitz when $i \in\langle 1, p\rangle$.

Assumption 2. By performing an inner adjustment for (71) as

$$
\dot{q}(t)-\underline{q}_{e}(t)=\underline{\mathbf{A}}_{e}\left(\mathbf{q}(t)-\underline{\mathbf{q}}_{e}(t)\right)+\sum_{i=1}^{p} \underline{\mathbf{A}}_{e i}\left(\mathbf{q}\left(t-\tau_{i}\right)-\underline{\mathbf{q}}_{e}\left(t-\tau_{i}\right)\right),
$$

it follows from (73)

$$
\underline{\dot{q}}_{e}(t)=\dot{q}(t)-\underline{\mathbf{A}}_{e} \mathbf{q}(t)-\sum_{i=1}^{p} \underline{\mathbf{A}}_{e i} \mathbf{q}\left(t-\tau_{i}\right)+\underline{\mathbf{A}}_{e} \underline{\mathbf{q}}_{e}(t)+\sum_{i=1}^{p} \underline{\mathbf{A}}_{e i} \underline{\mathbf{q}}_{e}\left(t-\tau_{i}\right),
$$

and substituting the autonomous part of (1) then

$$
\begin{aligned}
\dot{q}_{e}(t)= & \left.\left(\mathbf{A}-\left(\underline{\mathbf{A}}_{e}-\mathbf{J} \overline{\mathbf{C}}\right)\right) \mathbf{q}(t)\right)+\sum_{i=1}^{p}\left(\mathbf{A}_{i}-\left(\underline{\mathbf{A}}_{i}-\mathbf{J} \overline{\mathbf{C}}_{i}\right)\right) \mathbf{q}\left(t-\tau_{i}\right)+, \\
& +\underline{\mathbf{A}}_{e} \underline{\mathbf{q}}_{e}(t)+\sum_{i=1}^{p} \underline{\mathbf{A}}_{e} \underline{\mathbf{q}}_{e}\left(t-\tau_{i}\right), \\
= & (\mathbf{A}-\underline{\mathbf{A}}) \mathbf{q}(t)+\sum_{i=1}^{p}\left(\mathbf{A}_{i}-\underline{\mathbf{A}}_{i}\right) \mathbf{q}\left(t-\tau_{i}\right)+\mathbf{J} \overline{\mathbf{C}} \mathbf{q}(t)+, \\
& +\mathbf{J} \sum_{i=1}^{p} \overline{\mathbf{C}}_{i} \mathbf{q}\left(t-\tau_{i}\right)+\underline{\mathbf{A}}_{e} \underline{\mathbf{q}}_{e}(t)+\sum_{i=1}^{p} \underline{\mathbf{A}}_{e} \underline{\mathbf{q}}_{e}\left(t-\tau_{i}\right) .
\end{aligned}
$$

Thus, for $\overline{\mathbf{C}} \in \mathbb{R}_{+}^{m \times n}, \mathbf{q}(t), \mathbf{q}\left(t-\tau_{i}\right) \in \mathbb{R}_{+}^{n}$, the lower state estimate is nonnegative if $\mathbf{J} \in \mathbb{R}_{+}^{n \times m}$ is nonnegative and $\underline{\mathbf{A}}_{e}$ and all $\underline{\mathbf{A}}_{e i}$ are pure (strictly) Metzler and Hurwitz.

Theorem 3. Designing interval observer (64) and (65) to state estimation of uncertain strictly Metzlerian system (1) and (2), then matrices $\overline{\mathbf{A}}_{e}, \underline{\mathbf{A}}_{e} \in \mathbb{M}_{-+}^{n \times n}, \overline{\mathbf{A}}_{e}^{\diamond}, \underline{\mathbf{A}}_{e}^{\diamond} \in \mathbb{M}_{-+}^{n \times n}$ and the matrices $\left\{\overline{\mathbf{A}}_{e i}, \underline{\mathbf{A}}_{e i} \in \mathbb{M}_{-+}^{n \times n}, i=1, \ldots, p\right\}$ are strictly Metzler and Hurwitz if for given strictly Metzler matrices $\overline{\mathbf{A}}, \underline{\mathbf{A}} \in \mathbb{M}_{-+}^{n \times n},\left\{\overline{\mathbf{A}}_{i}, \underline{\mathbf{A}}_{i} \in \mathbb{M}_{-+}^{n \times n}, i=1, \ldots, p\right\}$ and for nonnegative matrices $\overline{\mathbf{C}}, \underline{\mathbf{C}} \in \mathbb{R}_{+}^{m \times n}, \quad\left\{\overline{\mathbf{C}}_{i}, \underline{\mathbf{C}}_{i} \in \mathbb{R}_{+}^{m \times n}, i=1, \ldots, p\right\}$ there exist positive definite diagonal matrices $\mathbf{P}, \mathbf{Q}, \mathbf{R}_{k} \in \mathbb{R}_{+}^{n \times n}$ such that for $l=1, \ldots, s, h=1, \ldots, n-1, k=1, \ldots, m$, $i=1, \ldots, p, \Delta=((1 \leftrightarrow n) / n)$,

$$
\begin{array}{r}
\mathbf{P} \succ 0, \mathbf{R}_{k}>0, \\
\mathbf{P} \overline{\mathbf{A}}(l, l)_{\Delta}-\sum_{k=1}^{m} \mathbf{R}_{k} \underline{\mathbf{C}}_{d k} \prec 0, \mathbf{P} \underline{\mathbf{A}}(l, l)_{\Delta}-\sum_{k=1}^{m} \mathbf{R}_{l k} \overline{\mathbf{C}}_{d k} \prec 0, \\
\mathbf{P L}^{h} \overline{\mathbf{A}}(l+h, l)_{\Delta} \mathbf{L}^{h \mathrm{~T}}-\sum_{k=1}^{m} \mathbf{R}_{k} \mathbf{L}^{h} \underline{\mathbf{C}}_{d k} \mathbf{L}^{h \mathrm{~T}} \succ 0, \\
\mathbf{P L}^{h} \underline{\mathbf{A}}(l+h, l)_{\Delta} \mathbf{L}^{h \mathrm{~T}}-\sum_{k=1}^{m} \mathbf{R}_{k} \mathbf{L}^{h} \overline{\mathbf{C}}_{d k} \mathbf{L}^{h \mathrm{~T}}>0,
\end{array}
$$




$$
\begin{aligned}
& \mathbf{P} \overline{\mathbf{A}}_{i}(l, l)_{\Delta}-\sum_{k=1}^{m} \mathbf{R}_{k} \underline{\mathbf{C}}_{d i k} \prec 0, \mathbf{P} \underline{\mathbf{A}}_{i}(l, l)_{\Delta}-\sum_{k=1}^{m} \mathbf{R}_{k} \overline{\mathbf{C}}_{d i k} \prec 0, \\
& \mathbf{P L}^{h} \overline{\mathbf{A}}_{i}(l+h, l)_{\Delta} \mathbf{L}^{h \mathrm{~T}}-\sum_{k=1}^{m} \mathbf{R}_{k} \mathbf{L}^{h} \underline{\mathbf{C}}_{d i k} \mathbf{L}^{h \mathrm{~T}}>0, \\
& \mathbf{P L}^{h} \underline{\mathbf{A}}_{i}(l+h, l)_{\Delta} \mathbf{L}^{h \mathrm{~T}}-\sum_{k=1}^{m} \mathbf{R}_{k} \mathbf{L}^{h} \overline{\mathbf{C}}_{d i k} \mathbf{L}^{h \mathrm{~T}}>0, \\
& {\left[\begin{array}{cccc}
\mathbf{P} \overline{\mathbf{A}}+\overline{\mathbf{A}}^{\mathrm{T}} \mathbf{P}-\sum_{k=1}^{m} \mathbf{R}_{k} \mathbf{l l}^{\mathrm{T}} \underline{\mathbf{C}}_{d k}-\sum_{k=1}^{m} \underline{\mathbf{C}}_{d k} \mathbf{l l}^{\mathrm{T}} \mathbf{R}_{k} & * & \cdots & * \\
\mathbf{P} \overline{\mathbf{A}}_{1}-\sum_{k=1}^{m} \mathbf{R}_{k} \mathbf{l l}^{\mathrm{T}} \underline{\mathbf{C}}_{d 1 k} & -\mathbf{Q} & \cdots & * \\
\vdots & \vdots & \ddots & \vdots \\
\mathbf{P} \overline{\mathbf{A}}_{p}-\sum_{k=1}^{m} \mathbf{R}_{k} \mathbf{l l}^{\mathrm{T}} \underline{\mathbf{C}}_{d p k} & \mathbf{0} & \cdots & -\mathbf{Q}
\end{array}\right] \prec 0,} \\
& {\left[\begin{array}{cccc}
\mathbf{P} \underline{\mathbf{A}}+\underline{\mathbf{A}} \mathbf{P}-\sum_{k=1}^{m} \mathbf{R}_{k} \mathbf{l l}^{\mathrm{T}} \overline{\mathbf{C}}_{d k}-\sum_{k=1}^{m} \overline{\mathbf{C}}_{d k} \mathbf{l l}^{\mathrm{T}} \mathbf{R}_{k} & * & \cdots & * \\
\mathbf{P} \underline{\mathbf{A}}_{1}-\sum_{k=1}^{m} \mathbf{R}_{k} \mathbf{l l}^{\mathrm{T}} \overline{\mathbf{C}}_{d 1 k} & -\mathbf{Q} & \cdots & * \\
\vdots & \vdots & \ddots & \vdots \\
\mathbf{P} \underline{\mathbf{A}}_{p}-\sum_{k=1}^{m} \mathbf{R}_{k} \mathbf{l l}^{\mathrm{T}} \overline{\mathbf{C}}_{d p k} & \mathbf{0} & \cdots & -\mathbf{Q}
\end{array}\right] \prec 0,}
\end{aligned}
$$

where

$$
\begin{aligned}
& \overline{\mathbf{A}}(l+h, l)_{\Lambda}=\operatorname{diag}\left[\bar{a}_{1+h, 1}, \ldots, \bar{a}_{n, n-h}, \bar{a}_{1, n-h+1}, \ldots, \bar{a}_{h n}\right], \\
& \underline{\mathbf{A}}(l+h, l)_{\Delta}=\operatorname{diag}\left[\underline{a}_{1+h, 1}, \ldots, \underline{a}_{n, n-h}, \underline{a}_{1, n-h+1}, \ldots, \underline{a}_{h n}\right], \\
& \overline{\mathbf{A}}_{i}(l+h, l)_{\Lambda}=\operatorname{diag}\left[\bar{a}_{i, 1+h, 1}, \ldots, \bar{a}_{i, n, n-h}, \bar{a}_{i, 1, n-h+1}, \ldots, \bar{a}_{i h n}\right], \\
& \underline{\mathbf{A}}_{i}(l+h, l)_{\Delta}=\operatorname{diag}\left[\underline{a}_{i, 1+h, 1}, \ldots, \underline{a}_{i, n, n-h}, \underline{a}_{i, 1, n-h+1}, \ldots, \underline{a}_{i h n}\right] .
\end{aligned}
$$

When these conditions are successfully met, (33) defines the rule to compute positive gain matrix $\mathbf{J} \in \mathbb{R}_{+}^{n \times m}$.

Proof. The above theorem can be verified by following the same arguments used in proofs of Theorem 1 and Theorem 2 and so the proof is omitted.

Design conditions defined by Theorem 3 are interval equivalent to those defined by Theorem 2. Modification of the arguments also proves that interval equivalency to that defined by Theorem 1 can be obtained from Theorem 3 omitting the set of inequalities (80)-(82). Of course, if a solution exists, in the last mention case it is guaranteed that only $\overline{\mathbf{A}}_{e}, \underline{\mathbf{A}}_{e}, \overline{\mathbf{A}}_{e}^{\diamond}, \underline{\mathbf{A}}_{e}^{\diamond} \in \mathbb{M}_{-+}^{n \times n}$ are strictly Metzler and Hurwitz.

Modification of the arguments for pure Metzler system matrices is possible to be applied for the Metzlerian interval observer design, when adapting adequately the ways presented in Corollary 1.

\section{Illustrative Example}

To illustrate the design feasibility introduced in this paper, the parameters of the considered strictly Metzlerian timedelay system (1) and (2) are given as follows:

$$
\begin{aligned}
& \mathbf{A}=\left[\begin{array}{ccc}
-0.053 & 0.351 & 0.349 \\
0.029 & -0.655 & 0.106 \\
0.028 & 0.098 & -0.651
\end{array}\right], \mathbf{C}=\left[\begin{array}{ccc}
0.99 & 0.81 & 0 \\
0 & 1.35 & 1.08
\end{array}\right], \\
& \mathbf{A}_{1}=\left[\begin{array}{ccc}
-0.260 & 2.060 & 1.550 \\
0.140 & -3.640 & 0.360 \\
0.200 & 0.780 & -2.550
\end{array}\right], \mathbf{C}_{1}=\left[\begin{array}{ccc}
1.10 & 0 & 0 \\
0 & 1.50 & 0
\end{array}\right] \text {, } \\
& \mathbf{A}_{2}=\left[\begin{array}{ccc}
-0.270 & 1.450 & 1.940 \\
0.150 & -2.910 & 0.700 \\
0.080 & 0.200 & -3.960
\end{array}\right], \mathbf{C}_{2}=\left[\begin{array}{ccc}
0 & 0.90 & 0 \\
0 & 0 & 1.20
\end{array}\right] \text {. }
\end{aligned}
$$

It is evident that $\mathbf{A}, \mathbf{A}_{1}$, and $\mathbf{A}_{2}$ are strictly Metzler and $\mathbf{C}$, $\mathbf{C}_{1}$, and $\mathbf{C}_{2}$ are nonnegative.

To solve the task defined by Theorem 2, the auxiliary parameters are constructed: 


$$
\begin{aligned}
\mathbf{T} & =\left[\begin{array}{ll}
\mathbf{0}^{\mathrm{T}} & 1 \\
\mathbf{I}_{2} & \mathbf{0}
\end{array}\right], \mathbf{l}^{\mathrm{T}}=\left[\begin{array}{lll}
1 & 1 & 1
\end{array}\right], \Delta=\frac{(1 \leftrightarrow 3)}{3}, \\
\mathbf{A}(l, l)_{\Delta} & =\operatorname{diag}[-0.053-0.655-0.651], \mathbf{C}_{d 1}=\operatorname{diag}[0.990 .810] \\
\mathbf{A}(l+1, l)_{\Delta} & =\operatorname{diag}[0.0290 .0980 .349], \mathbf{C}_{d 2}=\operatorname{diag}[01.351 .08] \\
\mathbf{A}(l+2, l)_{\Delta} & =\operatorname{diag}[0.0280 .35100 .1060] \\
\mathbf{A}_{1}(l, l)_{\Delta} & =\operatorname{diag}[-0.260-3.640-2.550], \mathbf{C}_{d 11}=\operatorname{diag}[1.1000] \\
\mathbf{A}_{1}(l+1, l)_{\Delta} & =\operatorname{diag}[0.1400 .7801 .550], \mathbf{C}_{d 12}=\operatorname{diag}[01.500] \\
\mathbf{A}_{1}(l+2, l)_{\Delta} & =\operatorname{diag}[0.2002 .0600 .360], \\
\mathbf{A}_{2}(l, l)_{\Delta} & =\operatorname{diag}[-0.270-2.910-3.960], \mathbf{C}_{d 21}=\operatorname{diag}[00.900] \\
\mathbf{A}_{2}(l+1, l)_{\Delta} & =\operatorname{diag}[0.1500 .2001 .940], \mathbf{C}_{d 22}=\operatorname{diag}[001.20] \\
\mathbf{A}_{2}(l+2, l)_{\Delta} & =\operatorname{diag}[0.0801 .4500 .700]
\end{aligned}
$$

Using the SeDuMi package [28] to solve the given set of LMIs (50)-(55), the LMI variables are as follows:

$$
\begin{aligned}
\mathbf{P} & =\operatorname{diag}\left[\begin{array}{lll}
0.1379 & 0.2111 & 0.2067
\end{array}\right], \\
\mathbf{Q} & =\operatorname{diag}\left[\begin{array}{lll}
2.2715 & 4.0152 & 4.6094
\end{array}\right], \\
\mathbf{R}_{1} & =\operatorname{diag}\left[\begin{array}{lll}
0.0350 & 0.0030 & 0.0029
\end{array}\right], \\
\mathbf{R}_{2} & =\operatorname{diag}\left[\begin{array}{lll}
0.0076 & 0.0146 & 0.0088
\end{array}\right]
\end{aligned}
$$

The positive observer gain matrix is computed using (33) as $\mathbf{j}_{1}=\left[\begin{array}{l}0.2541 \\ 0.0144 \\ 0.0140\end{array}\right], \mathbf{j}_{2}=\left[\begin{array}{l}0.0550 \\ 0.0693 \\ 0.0426\end{array}\right], \mathbf{J}=\left[\begin{array}{ll}0.2541 & 0.0550 \\ 0.0144 & 0.0693 \\ 0.0140 & 0.0426\end{array}\right],\|J\|=0.2628$

which implies strictly Metzler and Hurwitz matrices:

$$
\begin{aligned}
& \mathbf{A}_{e}=\left[\begin{array}{ccc}
-0.3046 & 0.0709 & 0.2896 \\
0.0147 & -0.7603 & 0.0311 \\
0.0141 & 0.0291 & -0.6970
\end{array}\right], \rho\left(\mathbf{A}_{e}\right)=\left\{\begin{array}{l}
-0.2914 \\
-0.6990 \\
-0.7715
\end{array}\right\}, \\
& \mathbf{A}_{e 1}=\left[\begin{array}{ccc}
-0.5395 & 1.9775 & 1.5500 \\
0.1242 & -3.7440 & 0.3600 \\
0.1846 & 0.7160 & -2.5500
\end{array}\right], \rho\left(\mathbf{A}_{e 1}\right)=\left\{\begin{array}{l}
-0.2985 \\
-2.5793 \\
-3.9558
\end{array}\right\}, \\
& \mathbf{A}_{e 2}=\left[\begin{array}{ccc}
-0.2700 & 1.2213 & 1.8740 \\
0.1500 & -2.9230 & 0.6168 \\
0.0800 & 0.1874 & -4.0112
\end{array}\right], \rho\left(\mathbf{A}_{e 2}\right)=\left\{\begin{array}{l}
-0.1532 \\
-2.9247 \\
-4.1263
\end{array}\right\}, \\
& \mathbf{A}_{e}^{\diamond}=\left[\begin{array}{ccc}
-1.1141 & 3.2696 & 3.7136 \\
0.2889 & -7.4272 & 1.0079 \\
0.2787 & 0.9325 & -7.2582
\end{array}\right], \rho\left(\mathbf{A}_{e 2}\right)=\left\{\begin{array}{l}
-0.7612 \\
-6.7226 \\
-8.3159
\end{array}\right\} .
\end{aligned}
$$


One can verify that solving the set of LMIs (50)-(55), then feasible solutions exist also when suppressing (53) and
(54) for $i=1, i=2$, or $i=1,2$. The obtained positive observer gains

$$
\begin{aligned}
\mathbf{J}^{\varnothing 1} & =\left[\begin{array}{ll}
0.2500 & 0.0595 \\
0.0139 & 0.0672 \\
0.0132 & 0.0414
\end{array}\right], \mathbf{J}^{\varnothing 2}=\left[\begin{array}{ll}
0.2656 & 0.0503 \\
0.0144 & 0.0663 \\
0.0135 & 0.0429
\end{array}\right], \mathbf{J}^{\varnothing 1,2}=\left[\begin{array}{ll}
0.2767 & 0.0482 \\
0.0149 & 0.0662 \\
0.0120 & 0.0428
\end{array}\right], \\
\left\|\mathbf{J}^{\varnothing 1}\right\| & =0.2598, \quad\left\|\mathbf{J}^{\varnothing 1}\right\|=0.2727, \quad\left\|\mathbf{J}^{\varnothing 1}\right\|=0.2829,
\end{aligned}
$$

force strictly Metzler and Hurwitz matrices $\mathbf{A}_{e}, \mathbf{A}_{e 1}, \mathbf{A}_{e 2}, \mathbf{A}_{e}^{\diamond}$ in all cases but with different matrix norm of $\mathbf{J}$. This gives possibility to adjust results to given system matrix parameters.

Consider for illustration that $\mathbf{A}$ is pure Metzler and defined in such a way that

$$
\mathbf{A}=\left[\begin{array}{ccc}
-0.053 & 0.351 & 0.349 \\
0.029 & -0.655 & 0.106 \\
0.028 & 0 & -0.651
\end{array}\right], \mathbf{A}(l+1, l)_{\Delta}=\operatorname{diag}\left[\begin{array}{lll}
0.029 & 0 & 0.349
\end{array}\right]
$$

To reflect the structure of $\mathbf{C}$, it is necessary to define structured matrix variables

$$
\begin{aligned}
\mathbf{R}_{1}=\operatorname{diag}\left[r_{11} r_{12} 0\right], \mathbf{R}_{1} & =\operatorname{diag}\left[r_{21} r_{22} 0\right], \\
r_{i j} & >0 \text { for } i, j=1,2 .
\end{aligned}
$$

Solving (50)-(55), defining such structured $\mathbf{R}_{1}, \mathbf{R}_{2}$, then the resulting LMI variables are as follows:

$$
\begin{aligned}
\mathbf{P} & =\operatorname{diag}\left[\begin{array}{lll}
0.1343 & 0.2012 & 0.1635
\end{array}\right], \\
\mathbf{Q} & =\operatorname{diag}\left[\begin{array}{lll}
2.2751 & 3.9058 & 4.3242
\end{array}\right], \\
\mathbf{R}_{1} & =\operatorname{diag}\left[\begin{array}{lll}
0.0346 & 0.0027 & 0
\end{array}\right], \\
\mathbf{R}_{2} & =\operatorname{diag}\left[\begin{array}{lll}
0.0075 & 0.0140 & 0
\end{array}\right],
\end{aligned}
$$

and they produce nonnegative observer gain matrix

$$
\mathbf{J}=\left[\begin{array}{cc}
0.2577 & 0.0558 \\
0.0135 & 0.0694 \\
0 & 0
\end{array}\right],\|\mathbf{J}\|=0.2652
$$

which implies pure Metzler and Hurwitz matrix

$$
\mathbf{A}_{e}=\left[\begin{array}{ccc}
-0.3081 & 0.0669 & 0.2887 \\
0.0156 & -0.7597 & 0.0310 \\
0.0280 & 0 & -0.6510
\end{array}\right], \rho\left(\mathbf{A}_{e}\right)=\left\{\begin{array}{c}
-0.2836 \\
-0.6742 \\
-0.7610
\end{array}\right\},
$$

and strictly Metzler and Hurwitz matrices $\mathbf{A}_{e 1}, \mathbf{A}_{e 2}, \mathbf{A}_{e}^{\diamond}$. A pure Metzler and Hurwitz structure of $\mathbf{A}_{e}$ and strictly Metzler and Hurwitz structures of $\mathbf{A}_{e 1}, \mathbf{A}_{e 2}, \mathbf{A}_{e}^{\diamond}$ are obtained also when suppressing (53) and (54) for $i=1, i=2$, or $i=$ 1,2 if $\mathbf{A}$ is in the last defined pure Metzler form.

Consider, for simplicity, that the above defined set of strictly Metzler matrices represents the upper bounds of system matrices of the uncertain Metzlerian time-delay system and in the same manner that the above-defined nonnegative set of $\mathbf{C}, \mathbf{C}_{1}, \mathbf{C}_{2}$ represents the upper bounds of output matrices, while the lover bounds are as follows:

$$
\begin{aligned}
& \underline{\mathbf{A}}=\left[\begin{array}{ccc}
-0.108 & 0.296 & 0.294 \\
0.029 & -0.710 & 0.051 \\
0.028 & 0.043 & -0.706
\end{array}\right], \underline{\mathbf{C}}=\left[\begin{array}{ccc}
0.81 & 0.54 & 0 \\
0 & 1.17 & 0.81
\end{array}\right] \text {, } \\
& \underline{\mathbf{A}}_{1}=\left[\begin{array}{ccc}
-0.510 & 1.810 & 1.300 \\
0.140 & -3.890 & 0.110 \\
0.200 & 0.530 & -2.800
\end{array}\right], \underline{\mathbf{C}}_{1}=\left[\begin{array}{ccc}
0.90 & 0 & 0 \\
0 & 1.30 & 0
\end{array}\right] \text {, } \\
& \underline{\mathbf{A}}_{2}=\left[\begin{array}{ccc}
-0.520 & 1.200 & 1.690 \\
0.150 & -3.160 & 0.450 \\
0.080 & 0.200 & -4.210
\end{array}\right], \underline{\mathbf{C}}_{2}=\left[\begin{array}{ccc}
0 & 0.60 & 0 \\
0 & 0 & 0.90
\end{array}\right] \text {. }
\end{aligned}
$$


Constructing analogously the auxiliary parameters reflecting lover bounds, the set of LMIs (76)-(84) is satisfied with the feasible LMI variables:

$$
\begin{aligned}
\mathbf{P} & =\operatorname{diag}\left[\begin{array}{lll}
0.1151 & 0.2189 & 0.2088
\end{array}\right], \\
\mathbf{Q} & =\operatorname{diag}\left[\begin{array}{lll}
2.2478 & 4.3768 & 4.7416
\end{array}\right], \\
\mathbf{R}_{1} & =\operatorname{diag}\left[\begin{array}{lll}
0.0302 & 0.0023 & 0.0023
\end{array}\right], \\
\mathbf{R}_{2} & =\operatorname{diag}\left[\begin{array}{lll}
0.0036 & 0.0070 & 0.0030
\end{array}\right],
\end{aligned}
$$

and the positive observer gain matrix is computed as follows:

$$
\mathbf{J}=\left[\begin{array}{ll}
0.2623 & 0.0311 \\
0.0106 & 0.0318 \\
0.0109 & 0.0146
\end{array}\right],\|\mathbf{J}\|=0.2648,
$$

which leads to stable strictly Metzler and Hurwitz matrices of the interval observer dynamics

$$
\begin{aligned}
& \overline{\mathbf{A}}_{e}=\left[\begin{array}{ccc}
-0.2654 & 0.1730 & 0.3238 \\
0.0204 & -0.6979 & 0.0803 \\
0.0192 & 0.0751 & -0.6628
\end{array}\right], \underline{\mathbf{A}}_{e}=\left[\begin{array}{ccc}
-0.3676 & 0.0416 & 0.2604 \\
0.0185 & -0.7615 & 0.0167 \\
0.0173 & 0.0145 & -0.7218
\end{array}\right], \\
& \overline{\mathbf{A}}_{e 1}=\left[\begin{array}{ccc}
-0.4960 & 2.0196 & 1.5500 \\
0.1305 & -3.6813 & 0.3600 \\
0.1902 & 0.7610 & -2.5500
\end{array}\right], \underline{\mathbf{A}}_{e 1}=\left[\begin{array}{ccc}
-0.7985 & 1.7634 & 1.3000 \\
0.1283 & -3.9377 & 0.1100 \\
0.1881 & 0.5081 & -2.8000
\end{array}\right] \text {, } \\
& \overline{\mathbf{A}}_{e 2}=\left[\begin{array}{ccc}
-0.2700 & 1.2926 & 1.9120 \\
0.1500 & -2.9164 & 0.6714 \\
0.0800 & 0.1935 & -3.9731
\end{array}\right], \underline{\mathbf{A}}_{e 2}=\left[\begin{array}{ccc}
-0.5200 & 0.9640 & 1.6527 \\
0.1500 & -3.1695 & 0.4119 \\
0.0800 & 0.1902 & -4.2275
\end{array}\right], \\
& \widehat{\mathbf{A}}_{e}^{\diamond}=\left[\begin{array}{ccc}
-1.0315 & 3.4852 & 3.7858 \\
0.3009 & -7.2956 & 1.1116 \\
0.2894 & 1.0296 & -7.1860
\end{array}\right], \underline{\mathbf{A}}_{e}^{\diamond}=\left[\begin{array}{ccc}
-1.6861 & 2.7689 & 3.2131 \\
0.2968 & -7.8687 & 0.5385 \\
0.2853 & 0.7128 & -7.7493
\end{array}\right] .
\end{aligned}
$$

It is evident that

$$
\underline{\mathbf{A}}_{e}<\overline{\mathbf{A}}_{e}, \underline{\mathbf{A}}_{e 1}<\overline{\mathbf{A}}_{e 1}, \underline{\mathbf{A}}_{e 2}<\overline{\mathbf{A}}_{e 2}, \underline{\mathbf{A}}_{e}^{\diamond}<\overline{\mathbf{A}}_{e}^{\diamond} .
$$

Analyzing the results, it is evident that a stable interval observer is designed to the uncertain time-delay Metzlerian system, that is, all the interval observer system matrices $\overline{\mathbf{A}}_{e}, \underline{\mathbf{A}}_{e}, \overline{\mathbf{A}}_{e 1}, \underline{\mathbf{A}}_{e 1}, \overline{\mathbf{A}}_{e 2}, \underline{\mathbf{A}}_{e 2}, \overline{\mathbf{A}}_{e}, \underline{\mathbf{A}}_{e}^{\diamond} \in \mathbb{M}_{-+}^{n \times n}$ are strictly Metzler and Hurwitz.

Thus, it is documented that, by solving problems defined by Theorem 2 and Theorem 3, the observers designed for time-delay Metzlerian systems and uncertain time-delay Metzlerian systems are asymptotically stable and, in the case of interval observer, nonnegativity of the lower state estimate vector is forced.

\section{Concluding Remarks}

A novel approach is presented in the paper to address the problem of effectively computing a positive observer gain that establishes the set of stable strictly Metzler observer matrices in interval Metzlerian time-delay observer design.
Based on strictly Metzler system matrices defining Metzlerian linear time-delay systems, the associated constraints related to desired strictly Metzler observer system matrices are formulated as a set of LMIs and, replenished by two extended Lyapunov matrix inequalities, force interval observer stability. It is supposed that all matrix variables associated with this LMI ensemble are positive definite and diagonal. As an added result, it is showed that if the timedelay interval Metzlerian observer error has a common quadratic Lyapunov function of the proposed type, all associated multidimensional observer system matrices are Metzler and Hurwitz. The results are presented for strictly Metzler set $\mathbb{M}_{-+}^{n \times n}$; however, a way of adaptation to acting on pure Metzler set $\mathbb{M}_{-+}^{n \times n}$ is also given.

The contribution, in particular through Theorem 2 and Theorem 3 to observers for time-delay Metzlerian systems and uncertain time-delay Metzlerian systems, makes clear that both problems are essentially the same and that the results obtained in one of these directions is translatable in the other one. Further research topics could be focused on the problems in directions when to provide solutions related to time-varying delays. 
The proposed approach provides a numerically reliable computational framework, as illustrated using the presented numerical example.

\section{Data Availability}

All data for reverification of the results presented are given in the article and the authors have no other data. Software tools for the solution are standard and their use should be known to the scientific community (MATLAB with related toolboxes).

\section{Conflicts of Interest}

The authors declare that there are no conflicts of interest regarding the publication of this paper.

\section{Acknowledgments}

The work presented in this paper was supported by VEGA, the Grant Agency of the Ministry of Education, and the Academy of Science of Slovak Republic, under Grant no. 1/ $0608 / 17$. This support is very gratefully acknowledged.

\section{References}

[1] H. Nikaido, Convex Structures and Economic Theory, Academic Press, New York, NY, USA, 1968.

[2] H. L. Smith, Monotone Dynamical Systems. An Introduction to the Theory of Competitive and Cooperative Systems, American Mathematical Society, Providence, RI, USA, 1995.

[3] N. K. Son and D. Hinrichsen, "Robust stability of positive continuous time systems," Numerical Functional Analysis and Optimization, vol. 17, no. 5-6, pp. 649-659, 1996.

[4] J. M. Carnicer, J. M. Peña, and R. A. Zalik, "Strictly totally positive systems," Journal of Approximation Theory, vol. 92, no. 3, pp. 411-441, 1998.

[5] O. Pastravanu and M. H. Matcovschi, “ $(M, \beta)$-stability of positive linear systems," Mathematical Problems in Engineering, vol. 2016, p. 11, Article ID 9605464, 2016.

[6] N. Dautrebande and G. Bastin, "Positive linear observers for positive linear systems,"' in Proceedings of European Control Conference ECC'99, Karlsruhe, Germany, 1999.

[7] M. Ait Rami and F. Tadeo, "Linear programming approach to impose positiveness in closed-loop and estimated states," in Proceedings of the 16th International Symposium on Mathematical Theory of Networks And Systems, pp. 2470-2477, Kyoto, Japan, 2006.

[8] B. Shafai and A. Oghbaee, "Positive quadratic stabilization of uncertain linear system," in Proceedings of the 2014 IEEE Multi-Conference on Systems and Control, pp. 1412-1417, Antibes, France, 2014.

[9] M. Koksal and M. E. Koksal, "Commutativity of cascade connected discrete-time linear time-varying systems," Transactions of the Institute of Measurement and Control, vol. 37, no. 5, pp. 615-622, 2015.

[10] X. Ding, Z. Xiang, and C. Yang, "Robust stabilization and disturbance rejection of positive systems with time-varying delays and actuator saturation," Mathematical Problems in Engineering, vol. 2015, p. 10, Article ID 125343, 2015.

[11] L. Farina and S. Rinaldi, Positive Linear Systems. Theory and Applications, John Wiley \& Sons, New York, NY, USA, 2000.
[12] M. E. Koksal, "Decomposition of a second-order linear timevarying differential system as the series connection of two first order commutative pairs," Open Mathematics, vol. 14, no. 1, pp. 693-704, 2016.

[13] D. Krokavec and A. Filasová, "LMI based principles in strictly Metzlerian systems control design," Mathematical Problems in Engineering, vol. 2018, Article ID 9590253, 14 pages, 2018.

[14] D. G. Luenberger, Introduction to Dynamic Systems. Theory, Models and Applications, John Wiley \& Sons, New York, NY, USA, 1979.

[15] J. L. Gouzé, A. Rapaport, and M. Z. Hadj-Sadok, "Interval observers for uncertain biological systems," Ecological Modelling, vol. 133, no. 1-2, pp. 45-56, 2000.

[16] Z. Shu, J. Lam, H. Gao, B. Du, and L. Wu, "Positive observers and dynamic output-feedback controllers for interval positive linear systems," IEEE Transactions on Circuits and Systems, vol. 55, no. 10, pp. 3209-3222, 2008.

[17] Y. Wang, D. M. Bevly, and R. Rajamani, "Interval observer design for LPV systems with parametric uncertainty," Automatica, vol. 60, no. 10, pp. 79-85, 2015.

[18] H. Ethabet, T. Raissi, M. Amairi, and M. Aoun, "Interval observers design for continuous-time linear switched systems," IFAC-PapersOnLine, vol. 50, no. 1, pp. 6259-6264, 2017.

[19] F. Mazenc and O. Bernard, "Interval observers for linear timeinvariant systems with disturbances," Automatica, vol. 47, no. 1, pp. 140-147, 2011.

[20] D. Krokavec and A. Filasová, "Interval observer design for uncertain linear continuous-time Metzlerian systems," in Proceedings of the 28th Mediterranean Conference on Control and Automation MED'20, pp. 1051-1056, Saint-Raphaël, France, 2020.

[21] M. Ait Rami, J. Jordan, and M. Schönlein, "Interval observers for linear systems with time-varying delays," in Proceedings of the 19th International Symposium on Mathematical Theory of Networks And Systems MTNS2010, pp. 75-79, Budapest, Hungary, 2010.

[22] Y. Zhao, L. Liao, X. Cai, and Y. Liu, "Stabilization design for uncertain linear positive systems with time-delay," in Proceedings of the Positive Systems. Theory And Applications (POSTA 2018), pp. 165-177, Springer Nature, Cham, Switzerland, 2019.

[23] A. Berman, M. Neumann, and R. Stern, Nonnegative Matrices in Dynamic Systems, John Wiley \& Sons, New York, NY, USA, 1989.

[24] M. Ait Rami, U. Helmke, and F. Tadeo, "Positive observation problem for linear time-delay positive systems," in Proceedings Of the 15th Mediterranean Conference On Control \& Automation MED2007, pp. 1-6, Athens, Greece, 2007.

[25] R. A. Horn and C. R. Johnson, Matrix Analysis, Cambridge University Press, New York, NY, USA, 2013.

[26] R. E. Skelton, T. Iwasaki, and K. M. Grigoriadis, A Unified Algebraic Approach to Control Design, Taylor \& Francis, London, UK, 1998.

[27] F. Blanchini, P. Colaneri, and M. E. Valcher, "Switched positive linear systems," Foundations and Trends in Systems and Control, vol. 2, no. 2, pp. 101-273, 2015.

[28] D. Peaucelle, D. Henrion, Y. Labit, and K. Taitz, User's Guide for SeDuMi Interface, LAAS-CNRS, Toulouse, France, 2002. 